\title{
Anomalously Weak Scattering in Metal-Semiconductor Multilayer Hyperbolic Metamaterials
}

\author{
Hao Shen, ${ }^{1,2}$ Dylan Lu, ${ }^{1}$ Bryan VanSaders, ${ }^{3}$ Jimmy J. Kan, ${ }^{4}$ Hongxing Xu, ${ }^{2}$ Eric E. Fullerton, ${ }^{1,4}$ and Zhaowei Liu ${ }^{1,3,4, *}$ \\ ${ }^{1}$ Department of Electrical and Computer Engineering, University of California, San Diego, \\ 9500 Gilman Drive, La Jolla, California 92093-0407, USA \\ ${ }^{2}$ Beijing National Laboratory for Condensed Matter Physics, Institute of Physics, \\ Chinese Academy of Sciences, Beijing 100190, China \\ ${ }^{3}$ Materials Science and Engineering, University of California, San Diego, 9500 Gilman Drive, La Jolla, \\ California 92093-0418, USA \\ ${ }^{4}$ Center for Magnetic Recording Research, University of California, San Diego, 9500 Gilman Drive, \\ La Jolla, California 92093-0401, USA
}

(Received 19 November 2014; published 29 May 2015)

\begin{abstract}
In contrast to strong plasmonic scattering from metal particles or structures in metal films, we show that patterns of arbitrary shape fabricated out of multilayer hyperbolic metamaterials become invisible within a chosen band of optical frequencies. This is due to anomalously weak scattering when the in-plane permittivity of the multilayer hyperbolic metamaterials is tuned to match with the surrounding medium. This new phenomenon is described theoretically and demonstrated experimentally by optical characterization of various patterns in Au-Si multilayer hyperbolic metamaterials. This anomalously weak scattering is insensitive to pattern sizes, shapes, and incident angles, and has potential applications in scattering crosssection engineering, optical encryption, low-observable conductive probes, and optoelectric devices.
\end{abstract}

DOI: 10.1103/PhysRevX.5.021021

Subject Areas: Metamaterials, Plasmonics

\section{INTRODUCTION}

Electromagnetic (EM) wave scattering is a ubiquitous phenomenon in systems with refractive index contrast between an obstacle and the surrounding material [1]. When illuminated by incident EM waves, electric charges in the obstacle oscillate in response to the excitation field, transferring energy by reradiation or absorption. The reradiation pattern and polarization strongly depends on the geometry and size of the obstacle as well as the wavelengthdependent optical properties of its constituent materials [2]. In the past century, the study of EM scattering by obstacles has yielded deep physical insight in the prediction of light transmission by atmospheric dust, interstellar particles, and colloidal metals [3-6], and practical applications in radar cross-section control in stealth technology and medical imaging $[7,8]$. Recent advances in plasmonics have revealed strongly dispersive scattering in metallic nanostructures by localized surface plasmon resonances, which have been widely explored and utilized for imaging and sensing [9-13]. Coupling between nanostructures introduces additional degrees of control for improving the scattering efficiency and directionality at the nanometer scale [14-20]. However, the fixed optical properties of naturally

\footnotetext{
* Corresponding author. zhaowei@ucsd.edu

Published by the American Physical Society under the terms of the Creative Commons Attribution 3.0 License. Further distribution of this work must maintain attribution to the author(s) and the published article's title, journal citation, and DOI.
}

existing metallic materials limit the range of performance and impose theoretical and practical restrictions.

Metamaterials, one type of artificial nanostructured composites, provide a new route for realizing either attenuated or enhanced plasmonic scattering with designable bandwidths and directions [19-34]. By engineering material composition in space following the invariance of Maxwell's equations, transformation optics and conformal mapping enable unprecedented control of light for the realization of cloaking [21-24]. Alternatively, the scattering cross section of an object can be significantly reduced by additional thin layers of plasmonic cover based on scattering cancellation schemes [25-28]. Metamaterials have also been shown to produce either EM-induced-transparency-like features [29-31] or superscattering [32-34] by exploiting coupled resonances in subwavelength objects. Special designs of single plasmonic structures incorporate both suppressed and highly resonant scattering responses in close frequency ranges $[35,36]$. Yet, the existing proposed schemes rely heavily on mode engineering with complex nanoscale geometry design and are extremely sensitive to fabrication imperfections. Moreover, the resonances in these designs are typically accompanied by strong material absorptions, further limiting the practical applications.

In this paper, we describe an approach where, instead of manipulating resonances, we control the scattering by engineering the material properties of anisotropic multilayerbased metamaterials. Multilayer hyperbolic metamaterials (HMMs) have recently drawn tremendous attention for providing a simple but effective platform for tunable material properties [37-43]. Exploiting the properties of HMMs, we 
demonstrate control of EM wave interaction with plasmonic structures by engineering the effective optical constants in Au-Si multilayer HMMs. In contrast to strong plasmonic scattering from pure metallic structures, patterns of various sizes ranging from deep-subwavelength to wavelength scale in Au-Si multilayer HMMs become invisible in a manner that is not achievable with natural metallic or high-index materials. Such anomalously weak scattering (AWS) from metamaterials comprised of conductive components is possible when the permittivity of the HMMs along the direction of the incident electric field impedance matches with the surrounding medium. At wavelengths away from the AWS, Au-Si HMMs present either metalliclike or dielectriclike scattering behavior. This exotic dispersive plasmonic scattering in HMMs can be applied to patterns of arbitrary geometry or size, and by changing the filling ratios of constituent materials, it is possible to tune AWS behavior to the desired optical wavelengths. We demonstrate this AWS experimentally by the optical characterization of various patterns in Au-Si HMMs, with corroborating full-wave numerical simulations. This stealth functionality in HMMs may lead to potential applications in optical encryption, noninvasive conductive probe designs, and invisible optoelectric devices.

\section{CONCEPT OF ANOMALOUSLY WEAK SCATTERING}

When EM plane waves propagate through a homogeneous medium $\left(\varepsilon_{1}\right)$, the scattering potential built by the presence of a subwavelength obstacle made of nonmagnetic materials with permittivity contrast scatters waves omnidirectionally. For an incident $z$-polarized plane wave (TE) propagating in the $x-y$ plane, the wave equation describing the resulting electric-field distribution as EM waves interact with the obstacle can be written in a general form as

$$
\frac{1}{\omega \mu_{y}} \frac{\partial^{2}}{\partial x^{2}} E_{z}+\frac{1}{\omega \mu_{x}} \frac{\partial^{2}}{\partial y^{2}} E_{z}+\omega \varepsilon_{z} E_{z}=0
$$

where $E_{z}$ denotes the total electric field along the $z$ direction, $\omega$ is the angular frequency in free space, and $\varepsilon_{i}$ and $\mu_{i}$ $(i=x, y, z)$ are permittivity and permeability of the constituent materials along different axes, respectively (see Supplemental Material for analysis of TM wave incidence [44]). For nonmagnetic materials, only the permittivity along the $z$ direction in the obstacle responds to the incident electric field, leading to perturbation of the original EM waves. The general analytical expression for the resulting total electric field in the far field is a summation of the incident and the scattered fields given as follows [1],

$$
\begin{aligned}
E_{z}(\mathbf{r}) \hat{z}= & E_{z}^{(i)}(\mathbf{r}) \hat{z}-k^{2} \hat{r} \times \hat{r} \times \hat{z} \\
& \times \int_{V}\left[\varepsilon_{z}\left(\mathbf{r}^{\prime}, \omega\right) / \varepsilon_{1}-1\right] E_{z}\left(\mathbf{r}^{\prime}\right) e^{-i \mathbf{k} \cdot \mathbf{r}^{\prime}} d \mathbf{r}^{\prime} \frac{e^{i k r}}{r},
\end{aligned}
$$

where $E_{z}^{(i)}$ denotes the incident electric field, $\varepsilon_{1}$ the permittivity of the surrounding media, the wave number $k=\omega \sqrt{\varepsilon_{1}} / c$, the position vector of the evaluating point $\mathbf{r}=r \cdot \hat{r}$, and the time-dependent factor $\exp (-i \omega t)$ has been omitted. This integration describes the overall effect on the scattering field evaluated at the far field by the scattering potential of the obstacle volume $(V)$ due to the permittivity contrast $\varepsilon_{z}\left(\mathbf{r}^{\prime}, \omega\right) / \varepsilon_{1}-1$. Therefore, $z$-polarized light scattering by the obstacle can be almost entirely eliminated by matching its permittivity along the $z$ direction with the surrounding medium; that is, $\varepsilon_{z}=\varepsilon_{1}$.

Unlike naturally occurring homogeneous metallic or dielectric materials that generally scatter light [Figs. 1(a) and 1(b)], the properties of multilayer-based metamaterials
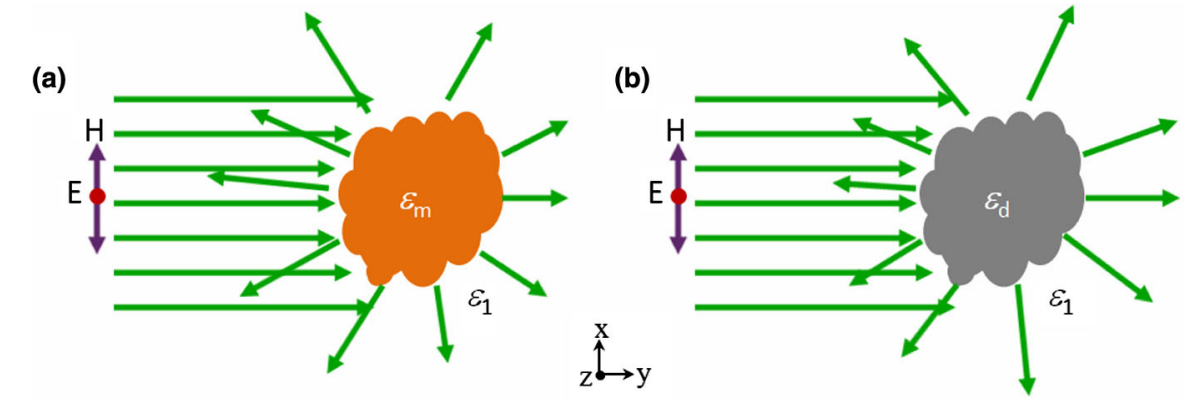

(c)

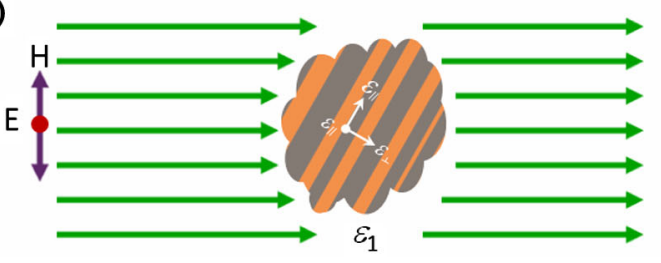

FIG. 1. Schematics of anomalously weak plasmonic scattering (AWS) by multilayer hyperbolic metamaterials (HMMs). Electromagnetic (EM) plane waves polarized along the $z$ axis are strongly scattered by an obstacle made of pure metal (a) or high-index dielectric (b) in the surrounding medium with permittivity $\varepsilon_{1}$, whereas AWS occurs for the obstacle composed of HMMs (c). 
can be readily engineered, enabling exotic EM wave interaction. The permittivity of HMMs can be made highly anisotropic, identified by the effective permittivities parallel $\left(\varepsilon_{\|}\right)$and perpendicular $\left(\varepsilon_{\perp}\right)$ to the plane of constituent layers [Fig. 1(c)]. By careful choice of constituent materials and layer thickness, light scattering by patterns in HMMs can be well controlled. In the case when the impedance matching condition $\varepsilon_{\|}=\varepsilon_{1}$ is satisfied, EM waves propagate through the medium without seeing the obstacle, achieving a minimum scattering cross section corresponding to AWS [Fig. 1(c)].

\section{NUMERICAL DEMONSTRATION OF ANOMALOUSLY WEAK SCATTERING}

To illustrate the concept of controlling EM wave scattering by metamaterials, we utilize multilayered gold and amorphous silicon composites in our study. As shown in Fig. 2(a), full-wave numerical studies of wave propagation through a single slit is carried out for pure $\mathrm{Au}, \mathrm{Si}$, and $\mathrm{Au}-\mathrm{Si} \mathrm{HMMs}$ with a metal filling ratio 0.6 (see Sec. VI for the full-wave simulations). All the slits are $100 \mathrm{~nm}$ thick, $300 \mathrm{~nm}$ wide, and have a surrounding medium with refractive index of 1.5. As indicated by the electric-field distribution, incident EM plane waves either reemit a cylindrical wave from the center of the slit in pure $\mathrm{Au}$ film or diffract at the edges in pure Si film. The resulting far-field patterns off homogeneous films are combinations of a Huygens point source (the slit) and plane waves (bulk transmission), which are attributed to their intrinsic optical properties, specifically, the negative permittivity of Au and large index of Si in the optical wavelengths (see Fig. S1 in the Supplemental Material for the permittivities of Au and Si [44]). However, EM waves propagate through a single slit in a Au-Si HMM by nearly retaining the original wave front with only mild attenuation and negligible Huygens point source behavior. Furthermore, this anomalous wave interaction is not sensitive to the incident angle [Fig. 2(a)(iv)], and thus, the intensity of the scattering in the AWS region is dramatically and uniformly decreased at all angles as long as TE polarization is maintained (see Fig. S2 [44] for the angular dependence of scattering at different wavelengths). When put together in an engineered manner, these originally strong-scattering constituent materials result in close to zero scattering cross section of the imbedded patterns.
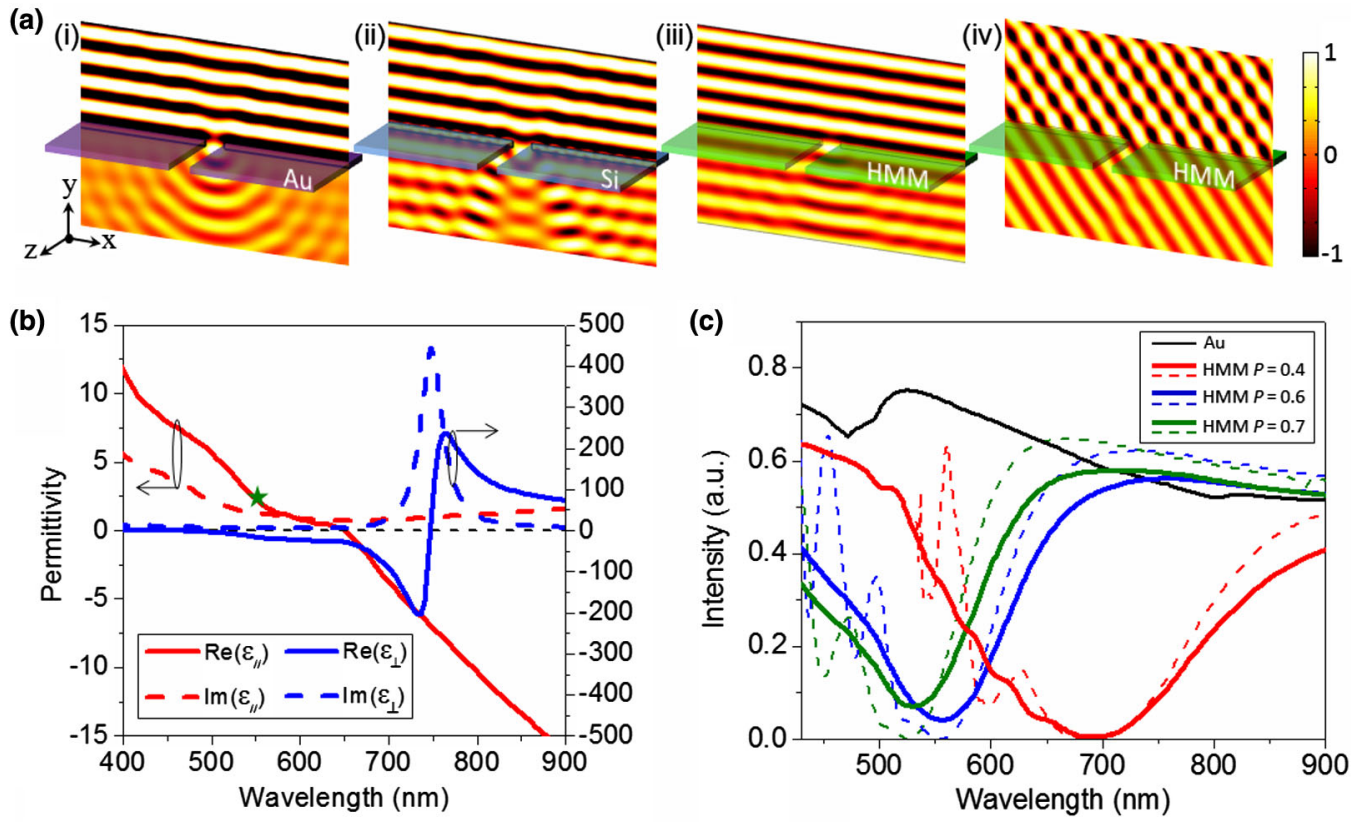

FIG. 2. Numerical simulations of plasmonic scattering by slits in HMMs. (a) Simulated electric-field distributions when a plane wave polarized perpendicular to the incident plane propagates through a single 300-nm-wide slit in pure Au film (i), Si film (ii), and Au-Si HMMs with Au filling ratio 0.6 (iii),(iv). Incident angles are $0^{\circ}$ (i)-(iii) and $53^{\circ}$ (iv). The thickness for all films is $100 \mathrm{~nm}$, and illumination wavelength is fixed at $550 \mathrm{~nm}$. (b) Real and imaginary parts of the permittivities for the Au-Si HMM with an Au filling ratio 0.6 perpendicular $\left(\varepsilon_{\perp}\right)$ and parallel $\left(\varepsilon_{\|}\right)$to the constituent layers are calculated based on the effective medium theory. The black dashed line corresponds to the zero line and the green star locates the permittivity parallel to the layer at the wavelength of $550 \mathrm{~nm}$. (c) Simulated scattering intensity in the forward direction for EM waves normally incident onto an 80-nm-wide slit in the Au-Si HMMs with a thickness of $100 \mathrm{~nm}$ when the Au filling ratio $P$ is tuned. Results for Au-Si HMMs assigned with complex (solid lines) or only real part (dashed lines) of the permittivities are presented. Scattering curves for the same slit in the pure Au film of the same thickness are included for comparison. 
The microscopic mechanism of the AWS phenomenon can be explained by the current distribution inside the Au-Si multilayer HMM [45]. The incident light generates opposite current directions in the Au layer and the Si layer, resulting in scattering suppression. Since the layer thickness in the HMM is much smaller than the probing wavelength, an effective-medium approximation is commonly used to describe the permittivities along distinct directions, that is, $\varepsilon_{\|}$and $\varepsilon_{\perp}$ (Ref. [45]), which gives the same simulation results as those based on real layered structure. Figure 2(b) shows the anisotropic effective permittivities for a uniform $\mathrm{Au}-\mathrm{Si} \mathrm{HMM}$ with the $\mathrm{Au}$ filling ratio 0.6 , where the red (blue) lines correspond to the component parallel (perpendicular) to the layer surfaces (see Sec. VI for the calculation of effective permittivities). The parallel permittivity displays a Drude-like dispersion that has positive values at short wavelengths and gradually decreases below zero as the wavelength is increased. At the location denoted by the green star, the real part of the parallel permittivity exactly matches the surrounding medium at a wavelength of $550 \mathrm{~nm}$. According to Eq. (2), such an impedance match along the parallel direction alone results in zero scattering fields for a $z$-polarized incident EM wave, and thus AWS occurs. This condition can be perfectly satisfied and the incident plane wave is completely undisturbed if only the real part of the permittivity is considered. The imaginary part of permittivity in metallic components results in the attenuated transmission through the HMMs [Fig. 2(a)(iii)].

The strongly dispersive permittivities in the $\mathrm{Au}-\mathrm{Si}$ HMMs give rise to highly varying scattering intensity across the whole optical regime [Fig. 2(c)]. The scattering intensity dips correspond to the AWS, which is invariant to TE incident angle and occurs for a wide range of surrounding-medium permittivities, even air, due to the large range of permittivity values covered by the parallel HMM component. At longer wavelengths, the HMM behaves more like a metallic film with stronger scattering intensity due to the effectively negative parallel permittivity, whereas at shorter wavelengths, it behaves as a dielectric film with an effectively positive parallel permittivity (see Fig. S3 [44]). By using different filling ratios for $\mathrm{Au}$ and $\mathrm{Si}$, the AWS wavelength as well as the whole dispersive scattering curve can be tuned to desired wavelengths [Fig. 2(c)]. As the Au filling ratio decreases, the AWS intensity gradually approaches the ideal limit with almost zero scattering at $P=0.4$.

\section{EXPERIMENTAL DEMONSTRATION OF ANOMALOUSLY WEAK SCATTERING}

In our experiment, the HMMs consisting of 10 pairs of 6-nm Au and 4-nm Si layers are prepared by sequential dc magnetron sputtering of $\mathrm{Si}$ and Au layers onto glass substrates at room temperature. Structures of several dimensions are milled into the multilayers by focused ion beam (see Sec. VI for the fabrication process). In Fig. 3(a), bright-field STEM images of cross-sectional views at different magnifications indicate a well-formed periodic lattice structure in the Au-Si multilayers with a highly conformal coating across the substrate. There is no accumulation of the film roughness throughout the growth direction, and the 10-nm-thickness periods are well achieved. The element mapping for silicon and gold verifies the multilayer structure and some minor diffusion of $\mathrm{Au}$ into $\mathrm{Si}$ [Fig. 3(b)]. The layer thicknesses and multilayer structure are also confirmed by $\mathrm{x}$-ray-reflectivity scans.

Figure 3(c) presents measured TE-polarized scattering spectra for different slit widths with the inset SEM image showing a 110-nm-wide sample (see Sec. VI for the optical characterization). Strongly dispersive plasmonic scattering is observed in all slits with AWS occurring near the wavelength of $550 \mathrm{~nm}$ and strong scattering intensity at both shorter and longer wavelengths. The scattering intensity at the AWS wavelength slightly increases with the slit width due to the nonideal impedance matching resulting from finite material absorption in the HMMs. Numerical simulations [Fig. 3(d)] reproduce the phenomenon observed in real multilayer structures under an incident angle of $53^{\circ}$. Although these results are limited to TE wave incidence, scattering in the same system by TM waves also displays attenuated scattering intensity near the AWS wavelength, however, with greater scattering due to the material response $\left(\varepsilon_{\perp}\right)$ to the probing electric-field component perpendicular to the layer surfaces (see Fig. S4 for the scattering measurement for TM wave incidence [44]).

Control over scattering of patterns in the HMMs by engineering material properties is not limited to single onedimensional slits but is universal for periodic diffraction gratings or even arbitrarily shaped structures. A diffraction grating (period $750 \mathrm{~nm}$, slit width $140 \mathrm{~nm}$ ) is inscribed by a focused ion beam into a $\mathrm{Au}-\mathrm{Si} \mathrm{HMM}$ with a $\mathrm{Au}$ filling ratio 0.6 [Fig. 4(a), SEM image]. The scattering characterization of this grating shows an extremely small diffraction efficiency in the optical spectrum (white curve), corresponding to a prominent dark region of the pseudocolorful diffractive pattern in the green wavelengths [Fig. 4(a), colorful background]. Unlike conventional optical gratings with broadband diffraction, strongly dispersive scattering HMM gratings may find applications in optical imaging and sensing by filtering out unwanted spectral information.

Finally, we demonstrate that AWS in HMMs enables the optoencryption of information of arbitrary shaped structures by rendering it undetectable at chosen wavelengths. The University of California, San Diego (UCSD) logo is milled into the Au-Si HMMs as an example of inscribed information [Fig. 4(b)]. Corresponding scattering images at three representative wavelengths are captured by a CCD camera using narrow bandpass optical wavelength filters [Figs. 4(b)(ii)-4(b)(iv), see Sec. VI for the image capturing process]. Scattering images at blue and red wavelengths 
(a)

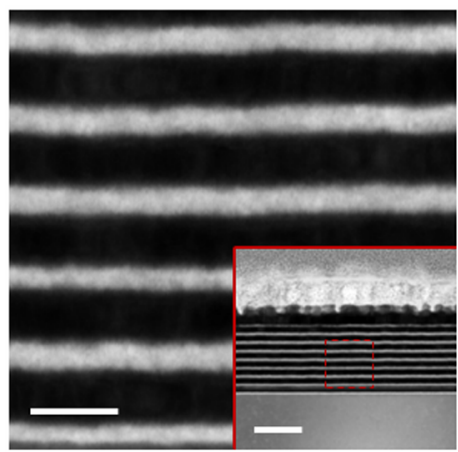

(b)

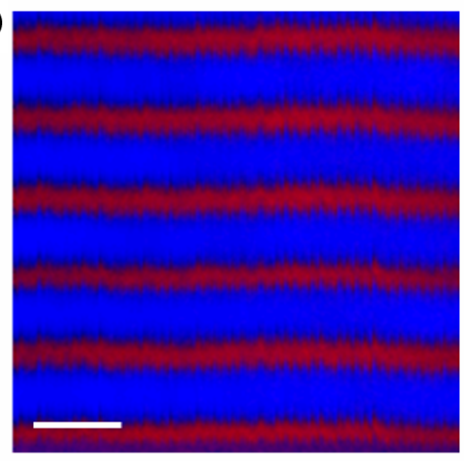

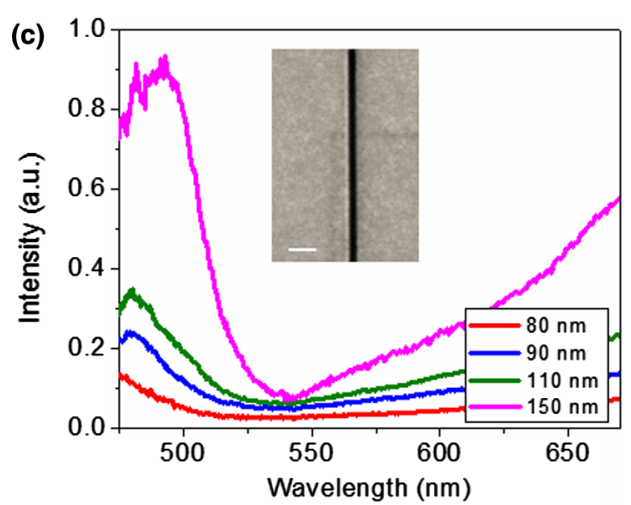

(d)

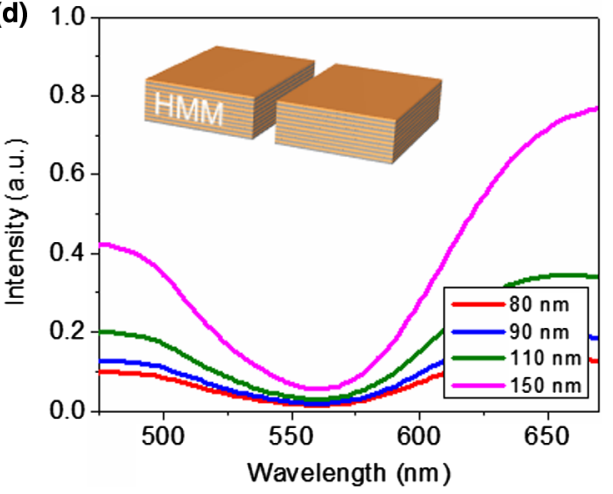

FIG. 3. Experimental demonstration of AWS by slits in Au-Si multilayer HMMs. (a) Bright-field STEM images for the fabricated Au$\mathrm{Si} \mathrm{HMM}$ with the white (black) area corresponding to the Si (Au) layer. The inset shows the STEM image at a smaller magnification for the whole cross section of the Au-Si multilayer with the top thick Pt capping for imaging purposes. The Au-Si multilayer is composed of 10 periods of $10 \mathrm{~nm}$ thickness each unit. The red dashed square outlines the imaging area of the main panel. Scale bars: main panel, $10 \mathrm{~nm}$; inset, $50 \mathrm{~nm}$. (b) Element mapping for constituent materials, silicon (red) and gold (blue), verifies the established multilayer lattice with some minor diffusion of Au into Si. Scale bar $10 \mathrm{~nm}$. (c) Measured dark-field scattering spectra in the forward direction for individual slits with different widths in the Au-Si HMM under TE-polarized light at an incident angle of 53 . The inset shows a SEM image of one fabricated slit with a width of $110 \mathrm{~nm}$. Scale bar $500 \mathrm{~nm}$. (d) Simulated scattering spectra in the forward direction for individual slits with different widths in the Au-Si HMM under TE-polarized light at an incident angle of 53 ${ }^{\circ}$. The inset plots the simulated geometry where real multilayered structure is used.

clearly outline the UCSD logo [Figs. 4(b)(ii) and 4(b)(iv)], whereas at the green wavelength, the image of the logo has a barely detectable intensity of $\sim 50$-fold dimmer due to AWS in the HMMs [Fig. 4(b)(iii)]. Such tailored information encryption in the HMMs for certain wavelengths can be applied in steganography and watermarking for anticounterfeiting purposes.

To demonstrate the robustness of AWS-enabled invisibility in the HMMs, distributed holes ( $190 \mathrm{~nm}$ diameter) forming UCSD letters are fabricated in the Au-Si HMMs [Fig. 5(a)], SEM image]. Scattering by holes displays similar spectral characteristics to slits, yet with a better spectral intensity contrast (see Fig. S5 for the scattering spectra of individual holes with different diameters [44]). By comparing forward dark-field scattering (red line) of hole features with the directional transmission (black line) through the uniform Au-Si HMMs (see Fig. S6 for measured transmission, reflection, and absorption through uniform Au-Si HMMs [44]), AWS by holes coincides with the maximum metamaterial transmission at the wavelength near $550 \mathrm{~nm}$ [Fig. 5(b)]. Corresponding UCSD letters in both the dark-field scattering [Fig. 5(c)(i)] and transmission [Fig. 5(c)(ii)] images become invisible at the AWS wavelength. Although the absolute transmission coefficient through the Au-Si HMM is moderate $(\sim 35 \%)$, the indiscernible intensity contrast between the transmission from the holes and the background through the HMM makes the hole patterns undetectable. However, this is not the case for wavelengths other than the AWS condition. At an exemplary wavelength of $735 \mathrm{~nm}$ in Fig. 5(d), the letters display well in both scattering [Fig. 5(d)(i)] and transmission [Fig. 5(d)(ii)] images. This is because scattering in all directions by holes dramatically increases [see Fig. 5(b)], which is a direct result of increased permittivity contrast between the air-filled hole space and surrounding HMM. Therefore, despite the absorption in HMMs, optical demonstration of the AWS reveals the potential capability of multilayer-based HMMs to conceal wavelength-scale features.

Although the performance of the current examples at visible frequencies is still limited by the considerable material absorption, the concept of AWS in near- or midinfrared regions with significantly reduced material losses 
(a)
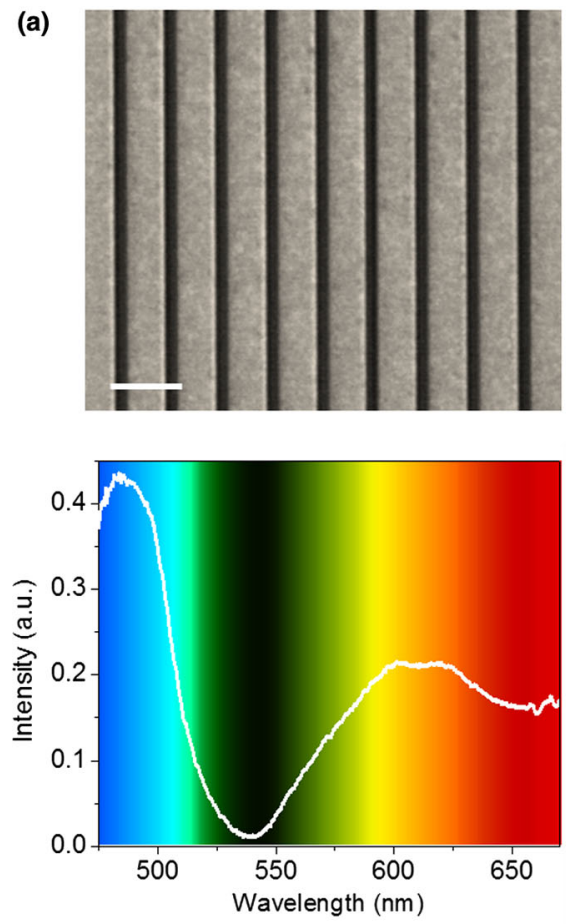

(b)
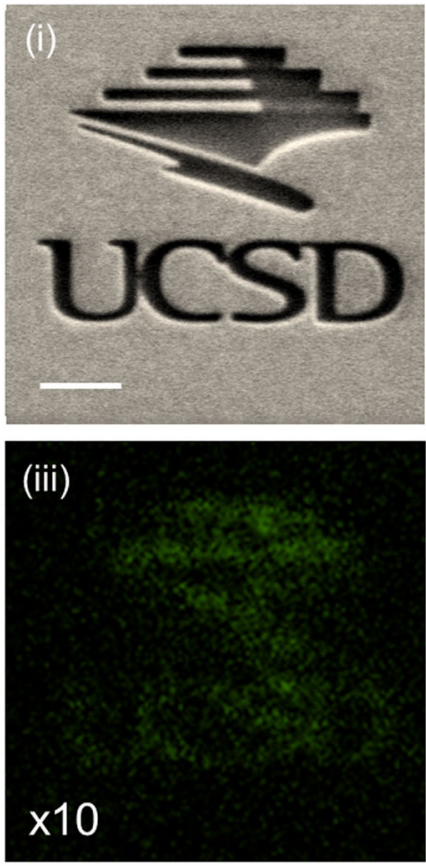

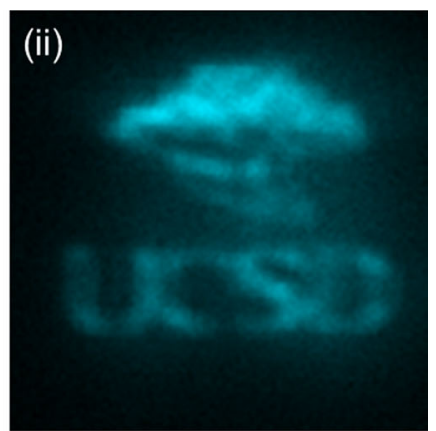

(iv)

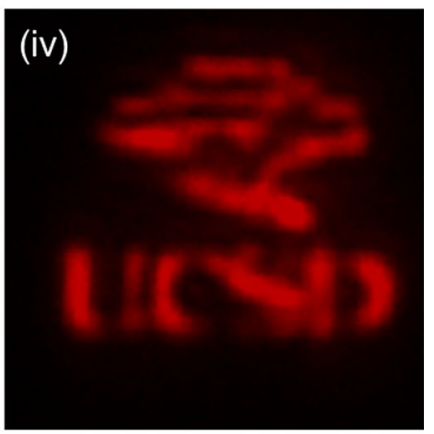

FIG. 4. Spectrum and imaging characterization of AWS by periodically and arbitrarily shaped patterns in Au-Si HMMs. (a) Top: SEM image of a HMM diffraction grating with a period of $750 \mathrm{~nm}$ and a slit width of $140 \mathrm{~nm}$ (Au fill ratio 0.6). Scale bar $1 \mu \mathrm{m}$. Bottom: Experimentally measured dark-field scattering spectrum in the forward direction for this type of grating (white line) with the background showing the same optical spectrum in pseudocolors with the intensity corresponding to the scattering signal. (b) SEM image of the UCSD logo in a Au-Si HMM with a Au filling ratio 0.6 (i). Scale bar is $1 \mu \mathrm{m}$. Dark-field scattering images in pseudocolors at the wavelengths $485 \mathrm{~nm}$ (ii), $540 \mathrm{~nm}$ (iii), and $735 \mathrm{~nm}$ (iv) are captured in the forward direction using bandpass optical wavelength filters. The image intensity at the green wavelength is multiplied by a factor of 10 for better comparison.

(a)
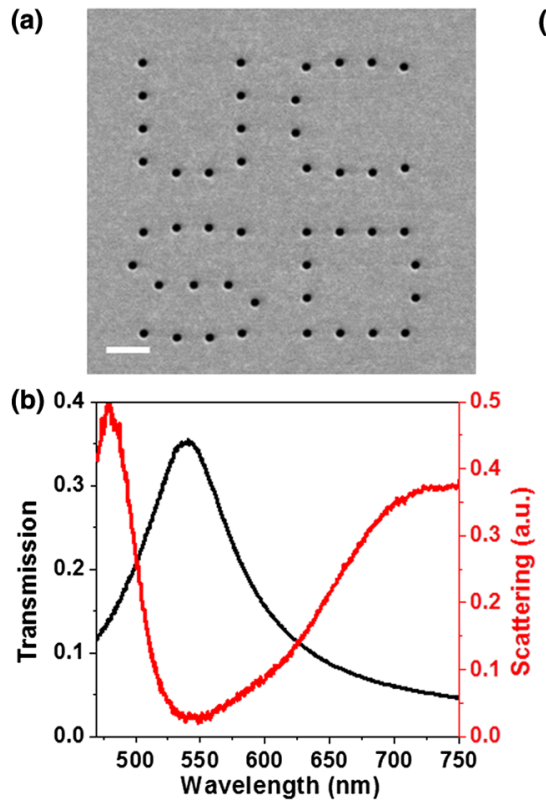

(c)
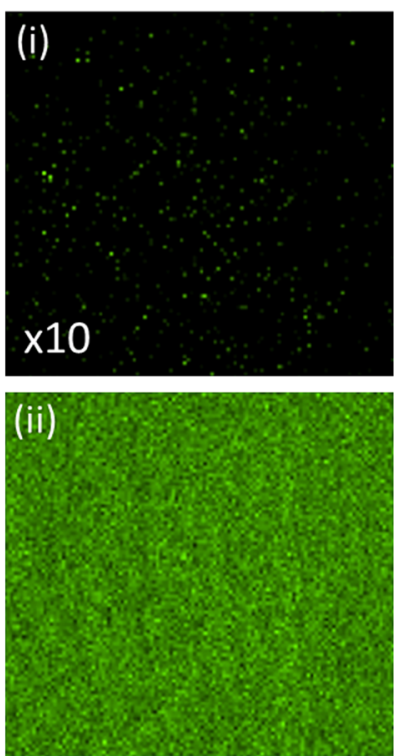

(d)
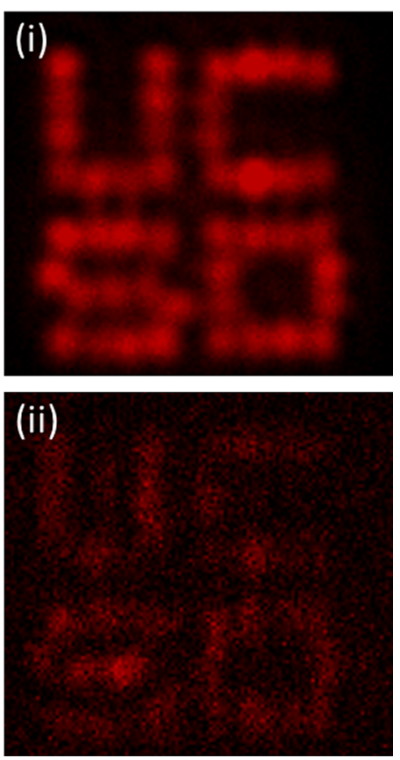

FIG. 5. Spectrum and imaging characterization of distributed holes in Au-Si HMMs. (a) SEM image of distributed holes forming letters UCSD in Au-Si HMMs with a Au filling ratio 0.6. Scale bar is $1 \mu \mathrm{m}$. (b) Transmission spectrum (black line) through the uniform $\mathrm{Au}-\mathrm{Si} \mathrm{HMMs}$ and forward dark-field scattering spectrum (red line) of distributed holes in Au-Si HMMs. (c) Forward dark-field scattering image (i) and corresponding transmission image under normal incidence (ii) in pseudocolor at the wavelength $540 \mathrm{~nm}$ (10 $\mathrm{nm}$ bandwidth). The image intensity in (i) is multiplied by a factor of 10 for better comparison. (d) Forward dark-field scattering image (i) and corresponding transmission image under normal incidence (ii) in pseudocolor at the wavelength $735 \mathrm{~nm}$ (10 nm bandwidth). 
may lead to various promising applications. For instance, such low-loss IR HMMs can be constructed by using very low filling ratio of metal and almost lossless semiconductors. In addition, the HMMs with nanowires embedded in a semiconductor host will further reduce the loss.

\section{CONCLUSIONS}

In conclusion, we demonstrate the control of optical scattering by engineering the material properties of Au-Si multilayer-based HMMs. Patterns in such HMMs become invisible in a chosen band of optical frequencies due to AWS when the permittivity of the constituent HMMs impedance matches with the surrounding medium. Experimental verifications of this new phenomenon by slits, holes, periodic gratings, and arbitrary objects in the HMMs prove this AWS insensitive to pattern size, shape, and incident angles. This geometry-independent effect in HMMs can be tuned to any desired wavelengths by varying the material filling ratios. Owing to the anisotropic material properties in multilayer HMMs, the control of scattering is dramatic only for TE polarization, which may be overcome by constructing isotropic three-dimensional metamaterials using a similar scheme. This new type of tunable HMMs provides an effective and versatile platform for controlling EM wave scattering cross sections, leading to new opportunities and potential applications in optical stealth and invisible optoelectric devices.

\section{METHODS}

\section{A. Electromagnetic simulations of scattering from slits}

Full-wave numerical simulation for the EM wave scattered by a single slit in a uniform film is carried out in a two-dimensional $x-y$ plane by using commercial software COMSOL MULTIPHYSICS ${ }^{\text {TM }}$. Plane waves with TE or TM polarizations are incident normally or at a chosen angle onto the slit. Light scattered $\left(\mathrm{k}_{\text {scattered }} \neq \mathrm{k}_{\text {incident }}\right)$ from the slits in the forward direction $\left(\mathrm{k}_{\text {scattered }}\right.$ less than $90^{\circ}$ changed from $\mathrm{k}_{\text {incident }}$ and within the transmission half-space defined by the uniform film boundary) is collected at different incident wavelengths to obtain the scattered intensity spectra. The permittivities for $\mathrm{Au}\left(\varepsilon_{m}\right)$ and $\mathrm{Si}\left(\varepsilon_{d}\right)$ are taken from Refs. [46,47], respectively, and plotted in Fig. S1 [44]. The material property for the Au-Si HMMs is described by the effective medium theory with the effective permittivity parallel to the layer $\varepsilon_{\|}=$ $P \varepsilon_{m}+(1-P) \varepsilon_{d}$ and that perpendicular to the layer $\varepsilon_{\perp}=\varepsilon_{m} \varepsilon_{d} /\left[P \varepsilon_{d}+(1-P) \varepsilon_{m}\right]$, where $P$ is the volumetric filling ratio of Au.

\section{B. Fabrication of Au-Si multilayer hyperbolic metamaterials}

The Au-Si multilayers are grown at room temperature under high vacuum by dc magnetron sputtering onto smooth glass substrates. The base pressure of the chamber is
$5 \times 10^{-8}$ Torr and the Ar sputtering gas pressure is fixed at 2.5 mTorr. Sputtering rates for Au at $50 \mathrm{~W}\left(\sim 2.5 \mathrm{~W} \mathrm{~cm}^{-2}\right)$ and $\mathrm{Si}$ at $100 \mathrm{~W}\left(\sim 5 \mathrm{~W} \mathrm{~cm}^{-2}\right)$ are 1.1 and $0.19 \AA \mathrm{As}^{-1}$, determined by x-ray-reflectivity measurements of calibration film sample thicknesses. Prior to $\mathrm{Au}$ and $\mathrm{Si}, 2 \mathrm{~nm}$ of Ta and $3 \mathrm{~nm}$ of Pd are deposited in order to promote adhesion of the multilayer structure to the glass substrate. The multilayer is composed of units of 4-nm-thick Si and 6-nm-thick Au with 10 periods in total. Patterns are inscribed into the multilayers by focused ion beam milling.

\section{Measurement of optical scattering spectra and images}

The optical scattering spectra are measured in a microspectroscopy system based on a Zeiss inverted microscope (Axio Observer D1m). Broadband light from a halogen lamp passes through a linear polarizer and an angular controllable condenser illuminates the sample at an incident angle of $53^{\circ}$ (N.A. $=0.8$ ). Dark-field scattering light from the samples in the forward direction is collected by an optical objective $(50 \times$ magnification, N.A. $=0.55)$ and analyzed by a Czerny-Turner spectrograph (Andor Shamrock 303i). The spectrograph is equipped with a diffraction grating of 150 lines $\mathrm{mm}^{-1}$ (blaze wavelength at $500 \mathrm{~nm}$ ) and a CCD camera (Andor Newton CCD). Direct transmission of the illumination light is carefully managed, so that it is not collected by the objective. The optical scattering images in Figs. 4(b), 5(c)(i), and 5(d)(i) are captured by the same microscope setup using a xenon lamp as illumination for its uniform intensity across the optical range, whereas transmission images in Figs. 5(c)(ii) and 5(d)(ii) are taken by a bright-field objective $(50 \times$ magnification, N.A. $=0.55)$ at normal incidence. After passing through a bandpass optical wavelength filter with a bandwidth of $20 \mathrm{~nm}$, the scattering and transmission images are taken by a CCD camera (Andor iXon EM + 897).

\section{ACKNOWLEDGMENTS}

The authors acknowledge financial support from the Office of Naval Research (ONR) Young Investigator Award (No. N00014-13-1-0535), DARPA Young Faculty Award (No. D13AP00054), NSF-CMMI (1120795), and ONR MURI program (N00014-13-1-0678). The authors thank P. Zhang for help with STEM characterization. Z. L. conceived the concept. D. L. performed numerical modeling and simulations in details. Z. L. and E. E. F. designed the materials, and J. J. K. and D. L. fabricated the samples. H. S. performed the optical characterizations. D. L. and H. S. prepared all of the figures. D. L., B. V. S., and Z. L. wrote the manuscript. All authors discussed the results and commented on the manuscript. Z. L. and E. E. F. supervised the project.

H. S. and D. L. contributed equally to this work. 
[1] M. Born and E. Wolf, Principles of Optics (Cambridge University Press, Cambridge, England, 1999).

[2] C. F. Bohren and D. R. Huffman, Absorption and Scattering of Light by Small Particles (Wiley, New York, 1998).

[3] G. Mie, Articles on the optical characteristics of turbid tubes, especially colloidal metal solutions, Ann. Phys. (Berlin) 330, 377 (1908).

[4] P. Debye, The heliograph of spheres of any material, Ann. Phys. (Berlin) 335, 57 (1909).

[5] H.C. v. d. Hulst, Light Scattering by Small Particles: Structure of Matter Series (Wiley, New York, 1957).

[6] H. M. Nussenzveig, Diffraction Effects in Semiclassical Scattering: Montroll Memorial Lecture Series in Mathematical Physics 1 (Cambridge University Press, Cambridge, England, 1992).

[7] E. F. Knott, J. F. Shaeffer, and M. T. Tuley, Radar Cross Section (Artech House, Boston, 1993).

[8] C. J. Morgan and W. R. Hendee, Introduction to Nuclear Magnetic Resonance Imaging (Multi-Media Publishing, Denver, CO, 1984).

[9] T. W. Ebbesen, H. J. Lezec, H. F. Ghaemi, T. Thio, and P. A. Wolff, Extraordinary optical transmission through subwavelength hole arrays, Nature (London) 391, 667 (1998).

[10] C. Genet and T. W. Ebbesen, Light in tiny holes, Nature (London) 445, 39 (2007).

[11] F. J. G. de Abajo, Colloquium: Light scattering by particle and hole arrays, Rev. Mod. Phys. 79, 1267 (2007).

[12] K. A. Willets and R. P. Van Duyne, Localized Surface plasmon resonance spectroscopy and sensing, Annu. Rev. Phys. Chem. 58, 267 (2007).

[13] J. N. Anker, W. P. Hall, O. Lyandres, N. C. Shah, J. Zhao, and R.P. Van Duyne, Biosensing with plasmonic nanosensors, Nat. Mater. 7, 442 (2008).

[14] E. Prodan, C. Radloff, N. J. Halas, and P. Nordlander, A hybridization model for the plasmon response of complex nanostructures, Science 302, 419 (2003).

[15] A. Kinkhabwala, Z. Yu, S. Fan, Y. AvlasevichK. Müllen, and W.E. Moerner, Large single-molecule fluorescence enhancements produced by a Bowtie Nanoantenna, Nat. Photonics 3, 654 (2009).

[16] J. A. Schuller, E. S. Barnard, W. Cai, Y. C. Jun, J. S. White, and M. L. Brongersma, Plasmonics for extreme light concentration and manipulation, Nat. Mater. 9, 193 (2010).

[17] L. Novotny and N. van Hulst, Antennas for light, Nat. Photonics 5, 83 (2011).

[18] H. A. Atwater and A. Polman, Plasmonics for improved photovoltaic devices, Nat. Mater. 9, 205 (2010).

[19] B. Luk'yanchuk, N. I. Zheludev, S. A. Maier, N. J. Halas, P. Nordlander, H. Giessen, and C. T. Chong, The Fano resonance in plasmonic nanostructures and metamaterials, Nat. Mater. 9, 707 (2010).

[20] J. A. Fan, C. Wu, K. Bao, J. Bao, R. Bardhan, N. J. Halas, V. N. Manoharan, P. Nordlander, G. Shvets, and F. Capasso, Self-assembled plasmonic nanoparticle clusters, Science 328, 1135 (2010).

[21] J. B. Pendry, D. Schurig, and D. R. Smith, Controlling electromagnetic fields, Science 312, 1780 (2006).

[22] U. Leonhardt, Optical conformal mapping, Science 312, 1777 (2006).
[23] D. Schurig, J. J. Mock, B. J. Justice, S. A. Cummer, J. B. Pendry, A. F. Starr, and D. R. Smith, Metamaterial electromagnetic cloak at microwave frequencies, Science 314, 977 (2006).

[24] J. Valentine, J. S. Li, T. Zentgraf, G. Bartal, and X. Zhang, An optical cloak made of dielectrics, Nat. Mater. 8, 568 (2009).

[25] A. Alu and N. Engheta, Achieving transparency with plasmonic and metamaterial coatings, Phys. Rev. E 72, 016623 (2005).

[26] A. Alu and N. Engheta, Multifrequency Optical Invisibility Cloak with Layered Plasmonic Shells, Phys. Rev. Lett. 100, 113901 (2008).

[27] P. Y. Fan, U. K. Chettiar, L. Y. Cao, F. Afshinmanesh, N. Engheta, and M. L. Brongersma, An invisible metalsemiconductor photodetector, Nat. Photonics 6, 380 (2012).

[28] V. A. Tamma, Y. H. Cui, and W. Park, Scattering reduction at near-infrared frequencies using plasmonic nanostructures, Opt. Express 21, 1041 (2013).

[29] N. Papasimakis, V. A. Fedotov, N. I. Zheludev, and S. L. Prosvirnin, Metamaterial Analog of Electromagnetically Induced Transparency, Phys. Rev. Lett. 101, 253903 (2008).

[30] S. Zhang, D. A. Genov, Y. Wang, M. Liu, and X. Zhang, Plasmon-Induced Transparency in Metamaterials, Phys. Rev. Lett. 101, 047401 (2008).

[31] N. Liu, L. Langguth, T. Weiss, J. Kastel, M. Fleischhauer, T. Pfau, and H. Giessen, Plasmonic analogue of electromagnetically induced transparency at the Drude damping limit, Nat. Mater. 8, 758 (2009).

[32] Z. C. Ruan and S. H. Fan, Superscattering of Light from Subwavelength Nanostructures, Phys. Rev. Lett. 105, 013901 (2010).

[33] Z. C. Ruan and S. H. Fan, Design of subwavelength superscattering nanospheres, Appl. Phys. Lett. 98, 043101 (2011).

[34] L. Verslegers, Z. F. Yu, Z. C. Ruan, P. B. Catrysse, and S. H. Fan, From Electromagnetically Induced Transparency to Superscattering with a Single Structure: A Coupled-Mode Theory for Doubly Resonant Structures, Phys. Rev. Lett. 108, 083902 (2012).

[35] F. Monticone, C. Argyropoulos, and A. Alu, Multilayered Plasmonic Covers for Comblike Scattering Response and Optical Tagging, Phys. Rev. Lett. 110, 113901 (2013).

[36] F. Monticone, C. Argyropoulos, and A. Alu, Layered plasmonic cloaks to tailor the optical scattering at the nanoscale, Sci. Rep. 2, 912 (2012).

[37] Z. Liu, H. Lee, Y. Xiong, C. Sun, and X. Zhang, Far-field optical hyperlens magnifying sub-diffraction-limited objects, Science 315, 1686 (2007).

[38] D. Lu and Z. Liu, Hyperlenses and metalenses for far-field super-resolution imaging, Nat. Commun. 3, 1205 (2012).

[39] Z. Jacob and V. M. Shalaev, Plasmonics goes quantum, Science 334, 463 (2011).

[40] H. N. S. Krishnamoorthy, Z. Jacob, E. Narimanov, I. Kretzschmar, and V. M. Menon, Topological transitions in metamaterials, Science 336, 205 (2012). 
[41] X. Yang, J. Yao, J. Rho, X. Yin, and X. Zhang, Experimental realization of three-dimensional indefinite cavities at the nanoscale with anomalous scaling laws, Nat. Photonics 6 , 450 (2012).

[42] N. Engheta, Pursuing near-zero response, Science 340, 286 (2013).

[43] D. Lu, J. J. Kan, E. E. Fullerton, and Z. Liu, Enhancing spontaneous emission rates of molecules using nanopatterned multilayer hyperbolic metamaterials, Nat. Nanotechnol. 9, 48 (2014).

[44] See Supplemental Material at http://link.aps.org/ supplemental/10.1103/PhysRevX.5.021021 for description of TM wave propagation in nonhomogeneous media and supplementary figures.

[45] S. A. Ramakrishna, J. B. Pendry, M. C. K. Wiltshire, and W. J. Stewart, Imaging the near field, J. Mod. Opt. 50, 1419 (2003).

[46] M. J. Weber, Handbook of Optical Materials (CRC Press, Boca Raton, 2003).

[47] D. E. Aspnes and A. A. Studna, Dielectric functions and optical parameters of Si, Ge, GaP, GaAs, GaSb, InP, InAs, and InSb from 1.5 to $6.0 \mathrm{eV}$, Phys. Rev. B 27, 985 (1983). 